\title{
Experimental and analytical studies on the response of 1/4-scale models of freestanding laboratory equipment subjected to strong earthquake shaking
}

\author{
Dimitrios Konstantinidis • Nicos Makris
}

Received: 13 May 2009 / Accepted: 8 May 2010 / Published online: 9 June 2010

(C) The Author(s) 2010. This article is published with open access at Springerlink.com

\begin{abstract}
This paper investigates the seismic response of freestanding equipment when subjected to strong earthquake motions ( $2 \%$ probability of being exceeded in 50 years). A two-step approach is followed because the displacement limitations of the shake table do not permit full-scale experiments. First, shake table tests are conducted on quarter-scale wooden block models of the equipment. The results are used to validate the commercially available dynamic simulation software Working Model 2D. Working Model is then used to compute the response of the full-scale freestanding equipment when subjected to strong, $2 \%$ in 50 years hazard motions. The response is dominated by sliding, with sliding displacements reaching up to $70 \mathrm{~cm}$. A physically motivated dimensionless intensity measure and the associated engineering demand parameter are identified with the help of dimensional analysis, and the results of the numerical simulations are used to obtain a relationship between the two that leads to ready-to-use fragility curves.
\end{abstract}

Keywords Laboratory equipment - Nonstructural components · Scaling · Rocking · Sliding · Fragility curves

D. Konstantinidis

Department of Civil and Environmental Engineering, University of California, Berkeley, CA, USA

D. Konstantinidis $(\varangle)$

Pacific Earthquake Engineering Research Center, Richmond Field Station, University of California, 1301 S. 46th Street, Richmond, CA 94804-4698, USA

e-mail: dakon@ce.berkeley.edu

N. Makris

Department of Civil Engineering, University of Patras, 26500 Patras, Greece

e-mail: nmakris@upatras.gr

N. Makris

Earthquake Engineering Research Center, University of California, Berkeley, CA, USA 


\section{Introduction}

Freestanding laboratory equipment on various floor levels of research laboratories, hospitals and other critical facilities may slide, rock, or even overturn during an earthquake. Rocking response is very sensitive to the geometry and mass distribution of the rocking object and the nature of the ground motion. Minor variations in the input can result in overturning (Yim et al. 1980; Makris and Roussos 2000; Makris and Konstantinidis 2003a). Therefore, rocking is in principle an undesirable response for the equipment since it is often the cause of mechanical damage or total loss in the event of overturning. Yet, even if overturning does not occur, the high acceleration spikes that develop during impact of the rocking equipment are a major concern, since they can result in damage of valuable scientific research material stored in the equipment by directly disturbing it or indirectly by causing failure in the electronic components of the equipment that maintain the controlled environment the material is stored in.

Sliding is the most favorable mode of response. Nonetheless, excessive sliding displacements may block a path or doorway that services evacuation or result in impact with walls or neighboring equipment. In practice, excessive sliding is prevented by restraining the equipment. Although this may succeed in reducing sliding displacements, it substantially amplifies accelerations (Konstantinidis and Makris 2005b). The problem of equipment sliding has been studied in the past at various scales by Shao and Tung (1999), Lopez Garcia and Soong (2003a,b), and Hutchinson and Chaudhuri (2006).

In an earlier paper (Konstantinidis and Makris 2009), the authors presented experimental and analytical studies on the seismic vulnerability of freestanding laboratory equipment located on various floor levels of a research laboratory building located at the University of California, Berkeley, campus-herein referred to as the UC Science Building. The equipment of interest included low-temperature refrigerators, freezers, incubators, and other heavy equipment. The study investigated the response of equipment to moderately strong motions (50 and 10\% probability of being exceeded in 50 years) which resulted in Peak Ground Displacements (PGD) or Peak Floor Displacements (PFD) that could be accommodated by the shake table at the Pacific Earthquake Engineering Research (PEER) Center, University of California, Berkeley. Shake table tests showed that there was no incidence of overturning due to excessive uplift. Uplift rotations ranged from very low, for two of the three specimens, to moderate, for the third, never exceeding $50 \%$ of the stockiness, $\alpha$ (the angle between a vertical line and the line that passes through the pivoting point and the center of mass of the equipment). For motions in this hazard level, the equipment tested exhibited excessive sliding displacements, reaching up to $60 \mathrm{~cm}$. The results of the tests were used to develop a dimensionless Engineering Demand Parameter (EDP) (a parameter that quantifies the response of the equipment), as a function of the Intensity Measure (IM) (a parameter of the excitation that corresponds to a certain seismic hazard level). Ready-to-use fraglity curves, which give the probability that the EDP will exceed a specific limit $c$, were generated.

The results of the shake table tests to moderate (POE of 50\% in 50 years and $10 \%$ in 50 years) hazard motions were used by Konstantinidis and Makris (2009) to validate the commercially available dynamic simulation software Working Model 2D (2000). However, experimental validation of Working Model for full-scale equipment to motions with large hazard level (POE of $2 \%$ in 50 years) could not be performed because the ground and floor displacements which resulted from these motions could not be accomodated by the shake table at the UC Berkeley PEER Center, which has a horizontal displacement capacity of $\pm 15 \mathrm{~cm}$. To address this challenge, shake table tests on quarter-scale wooden block models of the full-scale equipment prototypes were conducted. The reduction in length by a factor of 
4 corresponds to a reduction in time by a factor of $\sqrt{4}=2$. This can be shown by dimensional analysis for both sliding (Makris and Black 2003, 2004) and rocking motions (Makris and Konstantinidis 2003b), where accelerations are preserved in the model and the protoype, thereby tacitly accepting Froude similitude. The friction coeffient between the wooden block models and the shake table was considerably larger than the full-scale equipment to floor friction coefficient, thus resulting in rocking and overturning response rather than sliding. Simulations in Working Model using large friction coefficients validated the software's capability to capture the overturning behavior. The experimental validation of Working Model for sliding full-scale equipment and rocking wooden-block models together with a numerical validation for pure sliding and for pure rocking blocks provided confidence in its ability to estimate the response of equipment. In this study, Working Model is used to estimate the response of freestanding full-scale equipment to $2 \%$ in 50 years hazard level motions, and the results are used to generate ready-to-use fragility curves.

\section{Friction and shake table tests of wooden block models}

Three quarter-scale wooden blocks were made to model the three pieces of prototype equipment that were used for shake table tests presented in the earlier work published by the authors (2009). Figure 1 is a photograph of the three wooden block models resting on the shake table. The FORMA incubator prototype is also shown on the shake table, while the other two

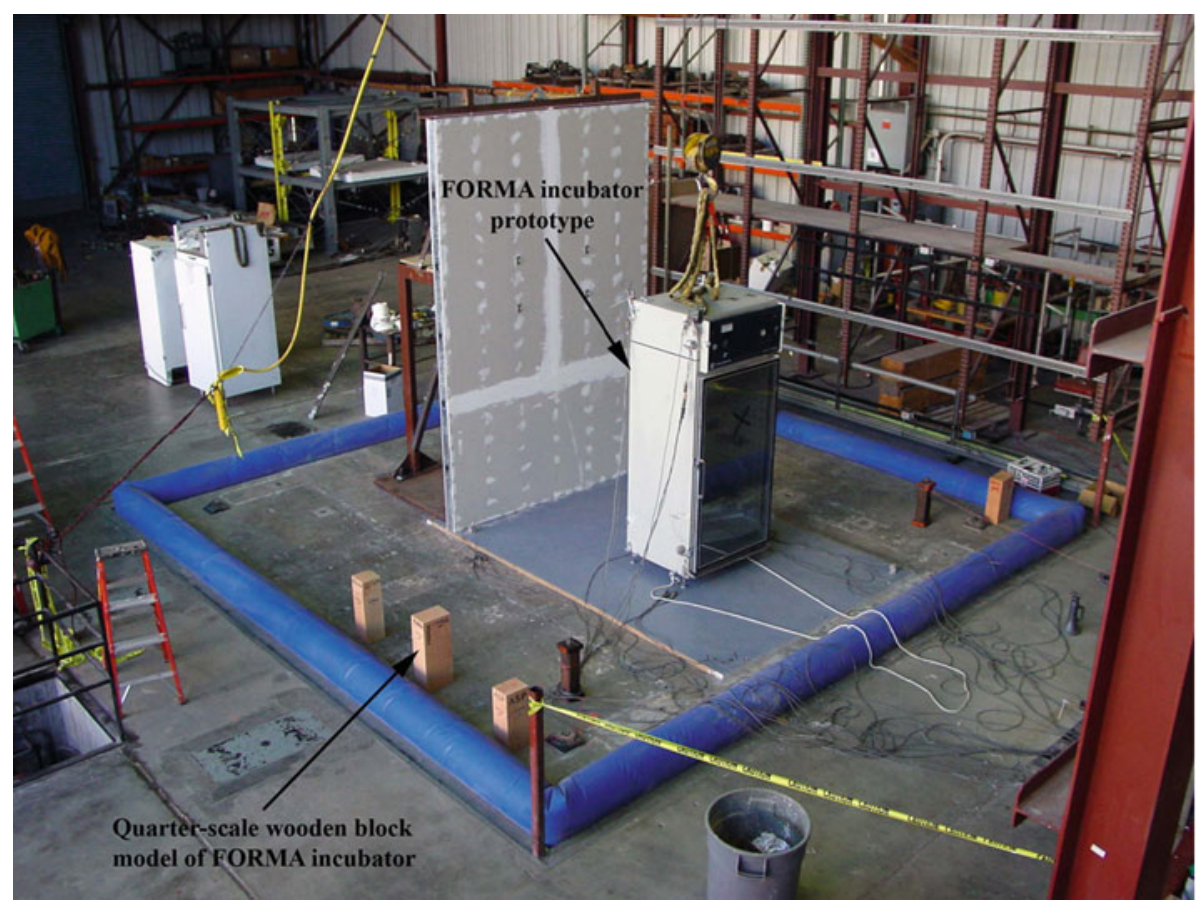

Fig. 1 The three 1/4-scale wooden block models together with the FORMA incubator prototype resting on the shake table at the UC Berkeley PEER Center. The other full-scale prototype equipment can be seen in the background 
Table 1 Geometric characteristics of the full-scale prototype equipment and the 1/4-scale wooden block models

\begin{tabular}{lclll}
\hline Equipment & Height $[\mathrm{cm}]$ & Width $[\mathrm{cm}]$ & $\alpha[\mathrm{rad}]$ & $p[\mathrm{rad} / \mathrm{s}]$ \\
\hline FORMA: prototype & 228.6 & 62.2 & 0.27 & 2.49 \\
FORMA: model & 57.2 & 15.6 & 0.27 & 4.98 \\
KELVINATOR: prototype & 213.4 & 66.0 & 0.30 & 2.57 \\
KELVINATOR: model & 53.4 & 16.5 & 0.27 & 5.14 \\
ASP: prototype & 180.3 & 58.4 & 0.31 & 2.79 \\
ASP: model & 40.1 & 14.6 & 0.27 & 5.58 \\
\hline
\end{tabular}

Test Specimen: wooden block model of FORMA incubator, Weight $=96 \mathbf{N}$

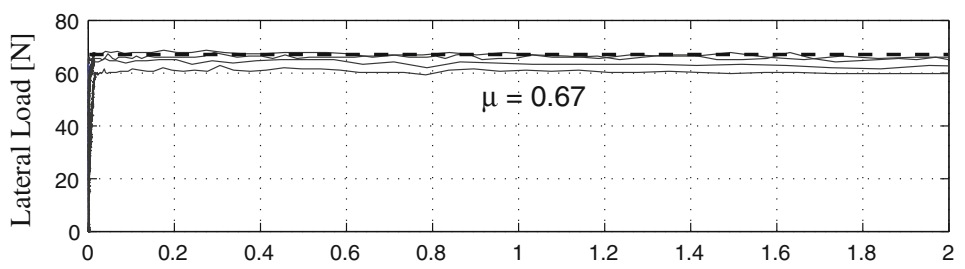

Test Specimen: wooden block model of KELVINATOR refrigerator, Weight $=\mathbf{6 8 N}$

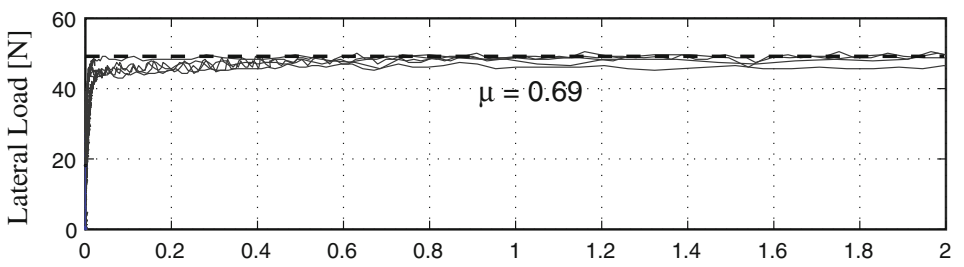

Test Specimen: wooden block model of $\mathbf{A S P}$ refrigerator, Weight $=\mathbf{5 8 N}$

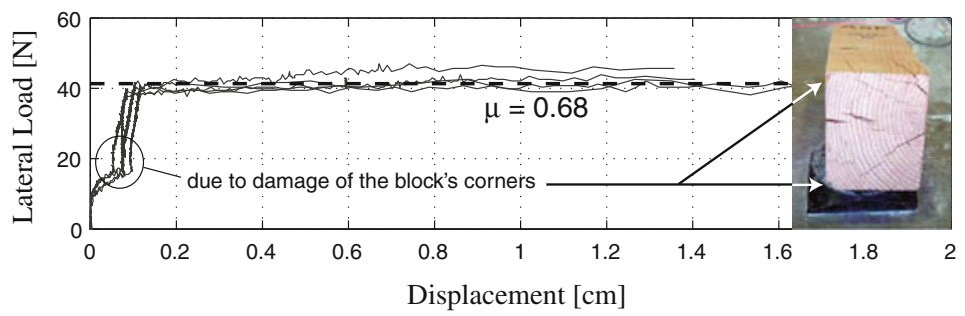

Fig. 2 Recorded load-displacement plots for the wooden block models obtained from quasi-static pull tests. The wood-concrete interface exhibits a nearly perfect rigid-plastic behavior

equipment prototypes (KELVINATOR and ASP refrigerators) can be seen in the background. Table 1 presents the geometrical characteristics of the full-scale prototype equipment and the quarter-scale wooden block models. Figure 2 shows results obtained from quasi-static pull tests on the wooden blocks. The behavior of the contact interface between the wooden blocks and the concrete surface of the shake table atop which the blocks rested is nearly rigid-plastic without exhibiting any difference between the static and kinetic values of the friction coefficient. The average value of the friction coefficient from the quasi-static pull tests on the three wooden blocks is about $\mu_{s}=\mu_{k}=0.68$. 
Table 2 Results of shake table tests on the wooden block models subjected to compressed $2 \%$ in 50 years Loma Prieta earthquake motions. Listed are also the outcomes predicted by Working Model simulations on full-scale prototypes subjected to the corresponding uncompressed motions

\begin{tabular}{|c|c|c|c|c|}
\hline $\begin{array}{l}\text { Equipment } \\
\text { prototype }\end{array}$ & $\begin{array}{l}\text { Compressed } \\
\text { earthquake } \\
\text { motion }\end{array}$ & PTA $[g]$ & $\begin{array}{l}\text { Shake table } \\
\text { experiment } \\
\text { outcome }\end{array}$ & $\begin{array}{l}\text { Working model } \\
\text { simulation outcome } \\
(\mu=0.68)\end{array}$ \\
\hline \multirow[t]{9}{*}{ FORMA } & Los Gatos PC, ground & 0.54 & Overturn & No overturn ${ }^{b}$ \\
\hline & Los Gatos PC, ground & 0.60 & Overturn & Overturn \\
\hline & Los Gatos PC FP, ground & 0.57 & Overturn & Overturn \\
\hline & Corralitos, ground & 0.84 & Overturn & No overturn ${ }^{\mathrm{b}}$ \\
\hline & Corralitos, ground & 0.83 & Overturn & Overturn \\
\hline & Gilroy Hist. Bldg., ground & 0.59 & Overturn & Overturn \\
\hline & Gilroy Hist. Bldg., ground & 0.58 & Overturn & No overturn ${ }^{\mathrm{a}}$ \\
\hline & Gilroy Hist. Bldg., ground & 0.59 & Overturn & No overturn ${ }^{\mathrm{b}}$ \\
\hline & Gilroy Hist. Bldg., 6th floor & 0.96 & Overturn & Overturn \\
\hline \multirow[t]{9}{*}{ KELVINATOR } & Los Gatos PC, ground & 0.54 & Overturn & Overturn \\
\hline & Los Gatos PC, ground & 0.60 & Overturn & Overturn \\
\hline & Los Gatos PC FP, ground & 0.57 & Overturn & Overturn \\
\hline & Corralitos, ground & 0.84 & No overturn & No overturn \\
\hline & Corralitos, ground & 0.83 & No overturn & No overturn \\
\hline & Gilroy Hist. Bldg., ground & 0.59 & Overturn & Overturn \\
\hline & Gilroy Hist. Bldg., ground & 0.58 & Overturn & Overturn \\
\hline & Gilroy Hist. Bldg., ground & 0.59 & Overturn & Overturn \\
\hline & Gilroy Hist. Bldg., 6th floor & 0.96 & Overturn & Overturn \\
\hline \multirow[t]{9}{*}{ ASP } & Los Gatos PC, ground & 0.54 & Overturn & No overturn \\
\hline & Los Gatos PC, ground & 0.60 & Overturn & Overturn \\
\hline & Los Gatos PC FP, ground & 0.57 & Overturn & No overturn \\
\hline & Corralitos, ground & 0.84 & Overturn & No overturn \\
\hline & Corralitos, ground & 0.83 & Overturn & No overturn \\
\hline & Gilroy Hist. Bldg., ground & 0.59 & Overturn & Overturn \\
\hline & Gilroy Hist. Bldg., ground & 0.58 & Overturn & Overturn \\
\hline & Gilroy Hist. Bldg., ground & 0.59 & Overturn & Overturn \\
\hline & Gilroy Hist. Bldg., 6th floor & 0.96 & Overturn & Overturn \\
\hline
\end{tabular}

a Although the block does not overturn, $\theta_{\max }$ nearly reaches $\alpha$

b Although the block does not overturn, $\theta_{\max }$ exceeds $\alpha$

Table 2 lists the compressed records that were used as input motions for the shake table tests on the wooden models together with the outcome of each experiment. The ground motions used are from a seismic hazard study for the UC Science site performed by Somerville (2001). The simulated floor motion used was obtained from a study by Lee and Mosalam (Comerio 2005; Lee and Mosalam 2005), who conducted dynamic analysis of a sophisticated structural model of the building. Since in most cases the wooden blocks overturned during the shake table tests, it was decided not to instrument the blocks but only to record whether the block survived the motion or overturned. More than one experiment were conducted for each 
motion in an effort to examine the repeatability of the outcomes; the outcomes were indeed repeatable in all but few cases.

\section{Validation of the Working Model software}

Since this study was part of a comprehensive study that employed the PEER-proposed Performance-Based Earthquake Engineering (PBEE) practical methodology, it was decided to utilize for the analysis a commercially available software; one that the practicing engineer could easily use to predict the response of laboratory equipment and other building contents alike. The shake table experiments presented in Konstantinidis and Makris (2009) indicated that the primary mode of response is sliding. However, rocking and possible overturning may happen for interfaces with larger coefficients of friction, $\mu$, and for equipment with more slender configurations ( $\operatorname{smaller} \alpha$ ). Therefore, the software of choice had to be able to capture both sliding and rocking response. Working Model 2D (2000) is a software that combines robust numerical techniques with sophisticated editing capabilities. Its main attraction is its capability to compute the motion of mechanically interacting rigid bodies under a variety of constraints and the action of time-varying forces.

One of the most challenging tasks in the dynamic simulation of rigid bodies is the treatment of the contact interfaces. In Working Model, the satisfaction of all imposed constraints at the contact interfaces is enforced simultaneously during the numerical integration. In the tangential direction, the contact interface of adjacent bodies is modeled by static and kinetic Coulomb friction (Working Model 2000). Regardless of whether there is sliding or not, the rigid body that models the equipment while engaging in rocking motion can impact the rigid body that models the ground. During an integration step, two colliding bodies may overlap by a small amount. In Working Model, collisions are detected by finding intersections between the geometries of bodies. Since the bodies are assumed rigid, for any two points on the body $\mathfrak{B},\left\|\mathbf{x}_{1}-\mathbf{x}_{2}\right\|=\left\|\mathbf{X}_{1}-\mathbf{X}_{2}\right\|$, for all time, where $\mathbf{x}$ is the one-to-one mapping $\mathbf{x}=\chi(\mathbf{X}, t)$, and $\mathbf{X}$ is the position in some reference configuration. This implies that the position and orientation of the edges of a rigid body are known for any time by tracking a master node. When intersection between edges is detected, Working Model computes forces sufficient to "repel" the bodies. Working Model employs an impulse-based collision model in which the coefficient of restitution is used (Working Model 2000).

The numerical integration of the equations of motion in conjunction with the satisfaction of the constraint conditions (friction and restitution), is done using a robust Kutta-Merson method (5th order Runge-Kutta). Integration error as well as model assembly and collision overlap tolerances can be set to achieve the desired precision. With the available variable-timestep Kutta-Merson scheme, near collision, the timestep is reduced appropriately to restrict the overlap between bodies from exceeding the specified overlap tolerance. For all the simulations presented in this report, the overlap error tolerance was set to $10^{-5} \mathrm{~cm}$.

\subsection{Validation for pure sliding}

We consider a rigid block resting on a base that is subjected to a horizontal excitation $\ddot{u}_{g}$. The interface coefficient of friction is $\mu$. An in-depth study of the response of a sliding mass on a moving base has been presented by Younis and Tadjbakhsh (1984). The solution to a constant acceleration pulse with amplitude $a_{p}$ and duration $T_{p}=2 \pi / \omega_{p}$ presented by Newmark (1965) is 


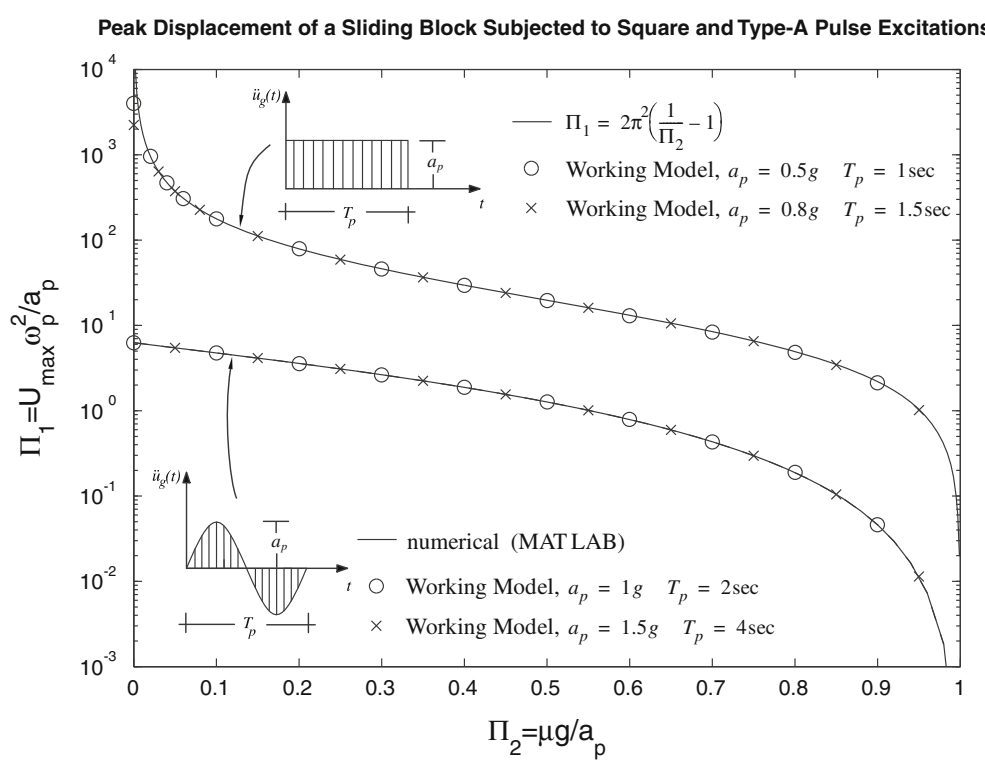

Fig. 3 Dimensionless displacement $\Pi_{1}$ as a function of the dimensionless strength $\Pi_{2}$ for rigid-plastic system subjected to rectangular and Type-A acceleration pulses with amplitude $a_{p}$ and duration $T_{p}=2 \pi / \omega_{p}$

$$
\frac{U_{\max } \omega_{p}^{2}}{a_{p}}=2 \pi^{2}\left(\frac{a_{p}}{\mu g}-1\right)
$$

where $U_{\max }$ is the maximum relative displacement of the block. Figure 3 plots with a solid line this dimensionless maximum relative displacement as a function of the dimensionless strength $\mu g / a_{p}$. The $\circ$ and $\times$ points plot the results obtained with the software Working Model where various combinations of the values of $a_{p}, T_{p}$, and $\mu$ have been used. Working Model captures with high fidelity the closed-form solution given by Eq. (1), which is plotted with a solid line.

Figure 3 also plots with a solid line the solution $U_{\max } \omega_{p}^{2} / a_{p}$ due to a Type-A (forwarddisplacement) pulse with acceleration amplitude $a_{p}$ and duration $T_{p}=2 \pi / \omega_{p}$ as a function of $\mu g / a_{p}$. The solution is obtained by numerically integrating in MATLAB (2002) the equation of motion associated with the Bouc-Wen model (Makris and Black 2003, 2004; Konstantinidis and Makris 2005a,b). The Bouc-Wen parameter values (Wen 1975, 1976), $\beta=\gamma=0.5, n=20$ and $u_{y}=10^{-5} \mathrm{~cm}$, were used to model the rigid-plastic behavior. Figure 3 shows that the Working Model solution is in excellent agreement with the solution obtained with MATLAB.

More evidence of the accuracy of the solutions obtained with Working Model is offered in Fig. 4 which plots the sliding response of a rigid mass subjected to three strong earthquakes. Note that despite the large variability in the peak values of the sliding displacements, the solutions obtained with Working Model are in excellent agreement with the MATLAB numerical solution.

\subsection{Validation for pure rocking}

In this section, we present a comparison of results obtained with Working Model to results obtained by numerically integrating the equation of motion for pure-rocking 
994 Northridge: Rinaldi 228
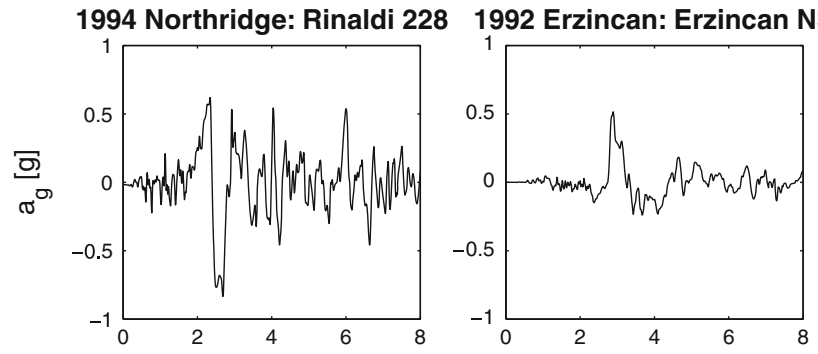

1995 Aigion: O.T.E. FP
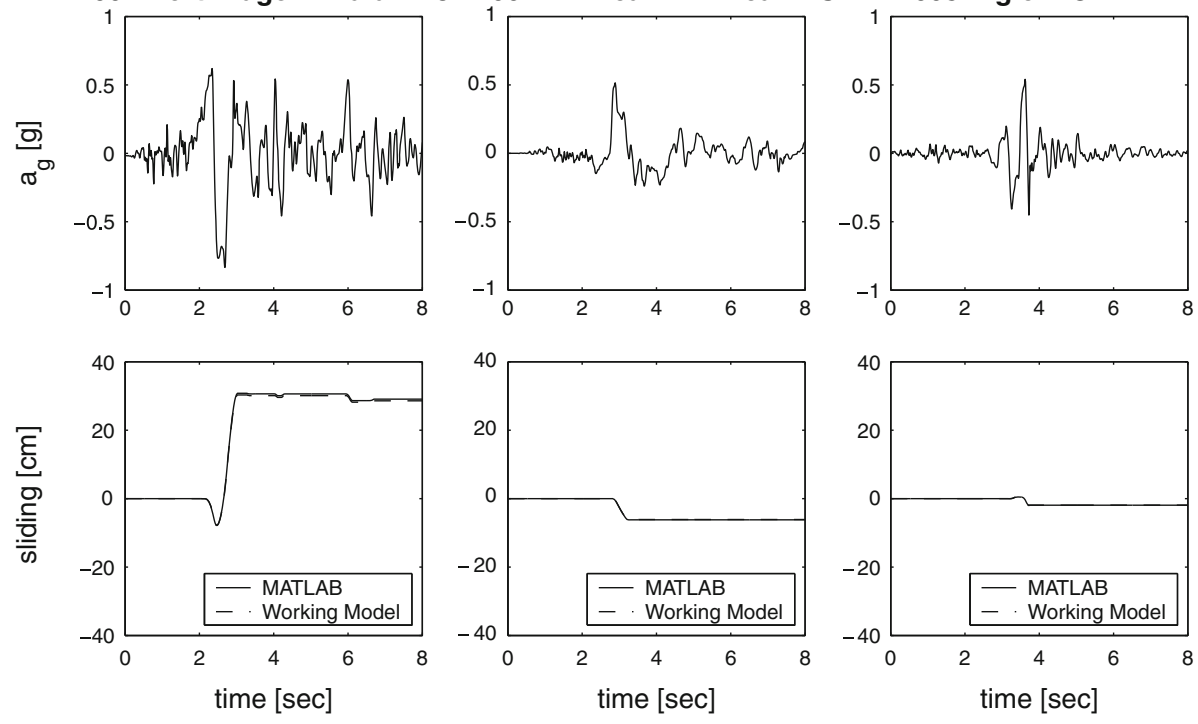

Fig. 4 Sliding displacement of a freestanding rigid mass $(\mu=0.3)$ subjected to three strong earthquakes. The response obtained with the software Working Model is in excellent agreement with the numerical solution obtained with MATLAB

(Makris and Roussos 2000; Makris and Konstantinidis 2003a), which yields time histories of the rotation and angular velocity of the rocking block. Standard ODE solvers available in MATLAB (2002) and a custom routine that detects impacts and imposes a reduction in angular velocity (to take into account energy lost upon impact) are used. Figure 5 plots the normalized rotation, $\theta / \alpha$, and angular velocity, $\dot{\theta} / p$, histories of a rigid block with frequency parameter $p=\sqrt{3 g / 4 R}=1.25 \mathrm{rad} / \mathrm{sec}$ (where $R$ is the distance from the pivot point to the center of mass of the block) and stockiness $\alpha=0.16$ rad when subjected to two strong ground motions. The solid line is the numerical solution obtained with MATLAB. The block survives the Rinaldi 228 motion (left) recorded during the 1994 Northridge, California, earthquake yet topples when subjected to the TCU052NS motion (right) recorded during the 1999 Chi-Chi, Taiwan, earthquake. The dashed line is the prediction of the software Working Model where the coefficient of friction has been set to a high value in order to avoid slipping. The solutions obtained with the two numerical codes are in excellent agreement.

Figure 6 plots the minimum overturning acceleration spectrum of the same block when subjected to a one-sine acceleration pulse (Type-A pulse) with acceleration amplitude $a_{p}$ and duration $T_{p}=2 \pi / \omega_{p}$. Notice the multivaluedness of $a_{p} / \alpha g$ that implies that a block can survive a pulse with acceleration amplitude larger than the minimum acceleration pulse necessary to overturn it. This interesting fact was first exposed by Zhang and Makris (2001). The shaded area in Fig. 6 is the unsafe region (i.e., where overturning occurs), while the blank area is the safe region (i.e., where overturning does not occur). Note that the software Working Model successfully predicts this multivaluedness of the response where a safe region appears above the minimum overturning acceleration line. The overall performance of Working Model is very good, with only minor degradation for shorter-period pulses $\left(\omega_{p} / p>10\right.$, or $\left.T_{p}<0.5 \mathrm{~s}\right)$ with large acceleration amplitudes $\left(a_{p} / \alpha g>15\right.$, or 

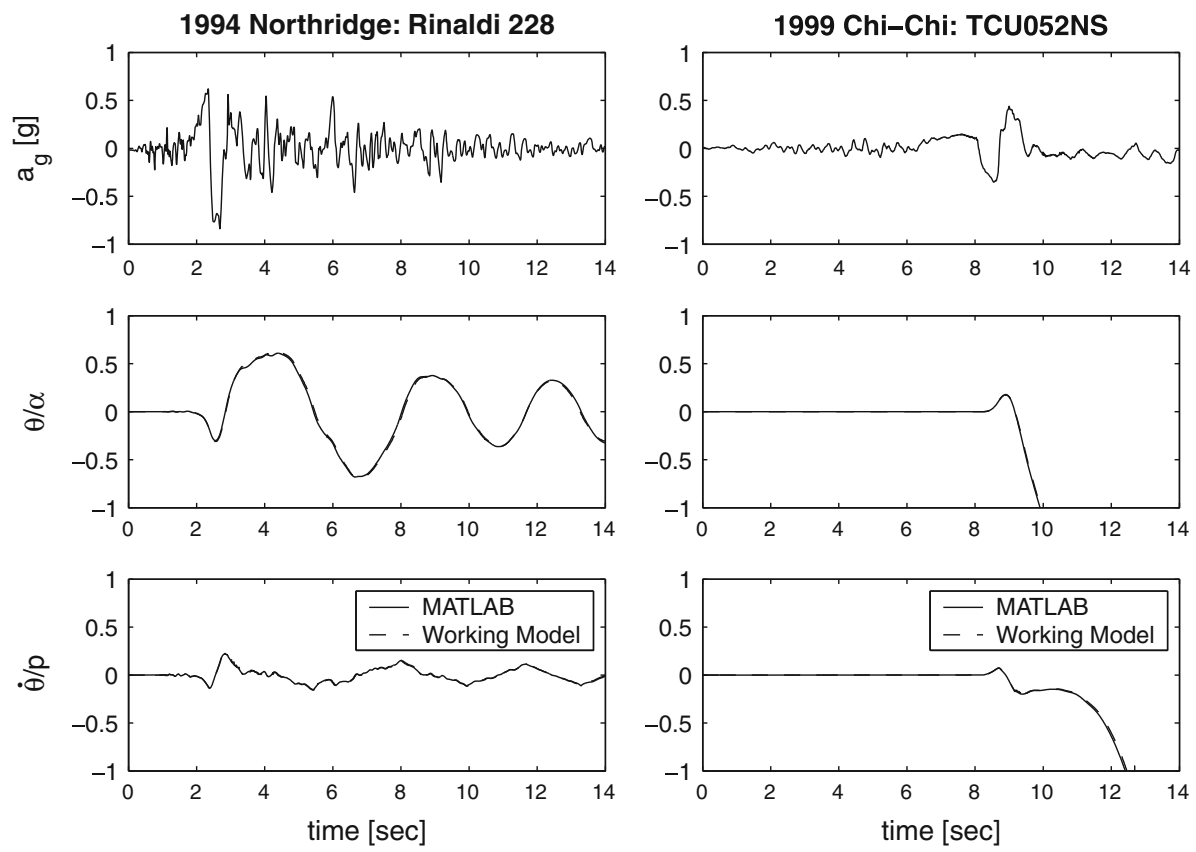

Fig. 5 Rotation and angular velocity of a rigid block with frequency parameter $p=1.25 \mathrm{rad} / \mathrm{s}$ and stockiness $\alpha=0.16$ rad subjected to two strong ground motions

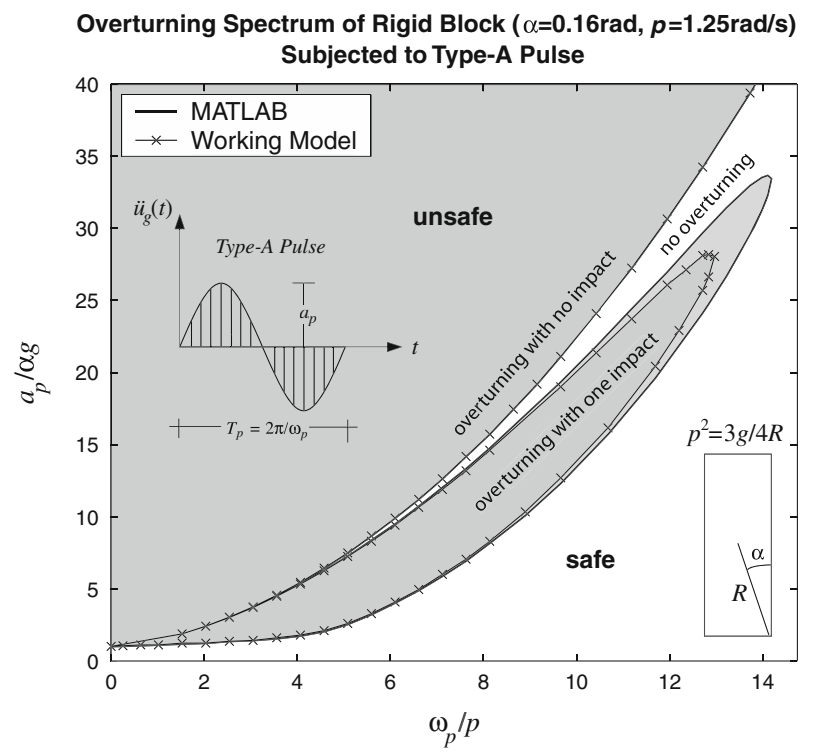

Fig. 6 Overturning acceleration spectrum of a rigid block with frequency parameter $p=1.25 \mathrm{rad} / \mathrm{s}$ and stockiness $\alpha=0.16$ rad subjected to a one-sine (Type-A) pulse 
$a_{p}>2.4 \mathrm{~g}$ ). More evidence on the fidelity of Working Model is offered in Konstantinidis and Makris (2005b).

\section{Comparison of results from working model simulations and shake table tests on quarter-scale wooden models}

The results of the shake table experiments that were carried on the quarter-scale wooden blocks (models) are of great value because they can validate the fidelity of results of numerical simulation studies conducted on the full-scale equipment (prototypes). Since the friction coefficients of the model-base interfaces were different from the friction coefficients of the prototype-base interfaces, two sets of simulation studies were conducted. First, the response of the full-scale prototype equipment was computed with Working Model by using a coefficient of friction at the sliding interface equal to $\mu_{s}=\mu_{k}=0.68$, the value obtained from the slow pull tests on the wooden blocks. The results of this analysis demonstrated that Working Model can capture well the experimentally observed overturning behavior of the wooden blocks. Then, once the fidelity of Working Model had been validated, the response of the full-scale prototype equipment was computed with the friction coefficients of the equipmentfloor sliding interfaces, presented in Konstantinidis and Makris (2009) and repeated here on the left column of Table 3 .

The bottom plot on the left column of Fig. 7 shows the time-compressed (by a factor of 2) acceleration history of the motion recorded at the Los Gatos Presentation Center during the 1989 Loma Prieta, California, earthquake. The plot above the acceleration history plots the resulting displacement history. The time-compressed motion was used as shake table input to test the quarter-scale wooden block model of the FORMA incubator. The final outcome of the experiment was that the wooden model overturned. The right-column plots of Fig. 7 show the response computed with Working Model for the full-scale FORMA incubator prototype subjected to the uncompressed motion. Note that the base displacement history of the uncompressed motion is 4 times larger in amplitude than the table displacement history of the compressed motion. The heavy solid lines of the top two windows on the right column of Fig. 7 plot the simulated sliding and uplift responses of the full-scale equipment with a coefficient of friction $\mu=0.68$, the value obtained from the quasi-static pull tests on the wooden blocks. While the simulated response predicts that the incubator does not overturn (as the experiment on the scaled model showed), the negative rotation $\theta$ of the equipment is so large that it in fact exceeds the stockiness value $\alpha$ at around $t=7.5 \mathrm{~s}$. Luckily, at the same time, the negative table acceleration which opposes the overturning of the equipment (positive $\theta$ is clockwise) marginally saves it from toppling.

This behavior whereby the equipment prototype marginally survives, while its corresponding wooden model overturns, is observed for a few of the motions. As shown in Table 2, when subjected to the Corralitos motion recorded during the 1989 Loma Prieta, California, earthquake, the wooden block overturns. The full-scale FORMA incubator, on the other hand, survives the motion. Figure 8 (right) which plots the response computed with Working Model, shows that around $t=5.5 \mathrm{~s}$ the rotation $\theta$ exceeds $\alpha$, and the equipment hangs on the verge of overturning; yet the restoring base acceleration spares the equipment. When the experiment was repeated on the shake table with the same motion but a slightly different Peak Table Acceleration $(0.83 g$ instead of $0.84 g$, due to imprecision of the shake table actuators), the wooden model overturned again. Interestingly, when the Working Model simulation was ran with PT A $=0.83 g$ (Peak Table Acceleration), the result was overturning. Except for these 
Table 3 Kinematic characteristics of the $2 \%$ in 50 years Loma Prieta motions used in this study. Together with the maximum computed equipment sliding displacement, $U_{\max }$, and interface friction coefficient, $\mu$, the motion PTA and $\omega_{p}=2 \pi / T_{p}$ produce the intensity measure, IM, and engineering demand parameter, EDP

\begin{tabular}{|c|c|c|c|c|c|c|}
\hline Equipment & Earthquake motion & $\begin{array}{l}T_{p}[\mathrm{~s}] \\
v_{p}[\mathrm{~cm} / \mathrm{s}]\end{array}$ & PTA $[\mathrm{g}]$ & $U_{\max }[\mathrm{cm}]$ & $\mathrm{IM}$ & EDP \\
\hline \multirow[t]{2}{*}{ FORMA } & Los Gatos PC, ground & $2.10,-75$ & 0.54 & 45.6 & 3.17 & 0.77 \\
\hline & Los Gatos PC, ground & $2.10,-75$ & 0.60 & 47.1 & 3.58 & 0.72 \\
\hline \multirow[t]{3}{*}{$\mu=0.23$ (slow pull) } & Los Gatos PC, ground & $2.10,-75$ & 0.57 & 40.3 & 3.39 & 0.64 \\
\hline & Corralitos, ground & $0.60,-61$ & 0.84 & 19.9 & 5.45 & 2.65 \\
\hline & Corralitos, ground & $0.60,-61$ & 0.83 & 20.6 & 5.38 & 2.78 \\
\hline \multirow[t]{4}{*}{$\mu=0.13$ (best fit) } & Gilroy Hist. Bldg., ground & $1.50,-84$ & 0.59 & 35.3 & 3.57 & 1.06 \\
\hline & Gilroy Hist. Bldg., ground & $1.50,-84$ & 0.58 & 35.9 & 3.43 & 1.12 \\
\hline & Gilroy Hist. Bldg., ground & $1.50,-84$ & 0.59 & 37.6 & 3.55 & 1.14 \\
\hline & Gilroy Hist. Bldg., 6th floor & $1.60,-120$ & 0.96 & 69.6 & 6.40 & 1.14 \\
\hline \multirow[t]{2}{*}{ KELVINATOR } & Los Gatos PC, ground & $2.10,-75$ & 0.54 & 31.7 & 2.19 & 0.53 \\
\hline & Los Gatos PC, ground & $2.10,-75$ & 0.60 & 34.1 & 2.50 & 0.52 \\
\hline \multirow[t]{3}{*}{$\mu=0.28($ slow pull $)$} & Los Gatos PC, ground & $2.10,-75$ & 0.57 & 32.8 & 2.36 & 0.52 \\
\hline & Corralitos, ground & $0.60,-61$ & 0.84 & 18.7 & 3.94 & 2.49 \\
\hline & Corralitos, ground & $0.60,-61$ & 0.83 & 18.8 & 3.88 & 2.53 \\
\hline \multirow[t]{4}{*}{$\mu=0.17$ (best fit) } & Gilroy Hist. Bldg., ground & $1.50,-84$ & 0.59 & 35.3 & 2.50 & 1.06 \\
\hline & Gilroy Hist. Bldg., ground & $1.50,-84$ & 0.58 & 38.2 & 2.39 & 1.19 \\
\hline & Gilroy Hist. Bldg., ground & $1.50,-84$ & 0.59 & 39.4 & 2.48 & 1.19 \\
\hline & Gilroy Hist. Bldg., 6th floor & $1.60,-120$ & 0.96 & 70.5 & 4.66 & 1.15 \\
\hline \multirow[t]{2}{*}{ ASP } & Los Gatos PC, ground & $2.10,-75$ & 0.54 & 28.8 & 1.71 & 0.49 \\
\hline & Los Gatos PC, ground & $2.10,-75$ & 0.60 & 30.6 & 1.98 & 0.47 \\
\hline \multirow[t]{3}{*}{$\mu=0.31($ slow pull $)$} & Los Gatos PC, ground & $2.10,-75$ & 0.57 & 28.8 & 1.86 & 0.48 \\
\hline & Corralitos, ground & $0.60,-61$ & 0.84 & 15.9 & 3.20 & 2.12 \\
\hline & Corralitos, ground & $0.60,-61$ & 0.83 & 16.8 & 3.15 & 2.26 \\
\hline \multirow[t]{4}{*}{$\mu=0.20$ (best fit) } & Gilroy Hist. Bldg., ground & $1.50,-84$ & 0.59 & 33.8 & 1.97 & 1.02 \\
\hline & Gilroy Hist. Bldg., ground & $1.50,-84$ & 0.58 & 34.3 & 1.88 & 1.07 \\
\hline & Gilroy Hist. Bldg., ground & $1.50,-84$ & 0.59 & 35.1 & 1.96 & 1.06 \\
\hline & Gilroy Hist. Bldg., 6th floor & $1.60,-120$ & 0.96 & 64.0 & 3.81 & 1.05 \\
\hline
\end{tabular}

marginal cases, Working Model managed to capture well the overturning of the wooden models.

Working Model also predicted correctly the two cases when the wooden model does not overturn. Figure 9 shows the experimental outcome (left) of the wooden block model of the KELVINATOR refrigerator subjected to the compressed Corralitos record of the 1989 Loma Prieta, California, earthquake and the computed response of the full-scale prototype with $\mu=0.68$ to the uncompressed record (right). The response computed with Working Model verifies that the block survives the motion.

Although they are presented in Table 2 for completeness, the Working Model simulations for the ASP refrigerator cannot be compared to the shake table experimental outcomes of 
equipment: FORMA incubator

motion: Loma Prieta, Los Gatos PC, FP GROUND (2\% in 50 years)

Working Model 2D simulation legend:

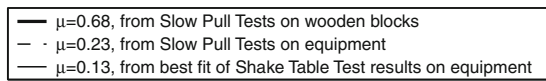

Quarter-Scale Wooden Block (Model)

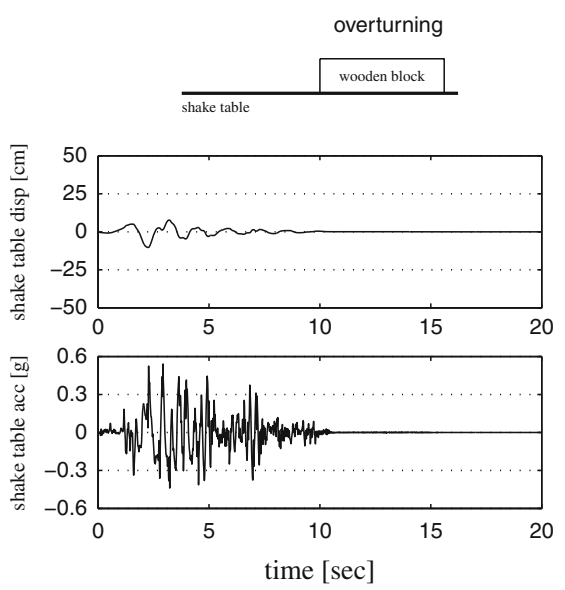

Full-Scale Equipment (Prototype)
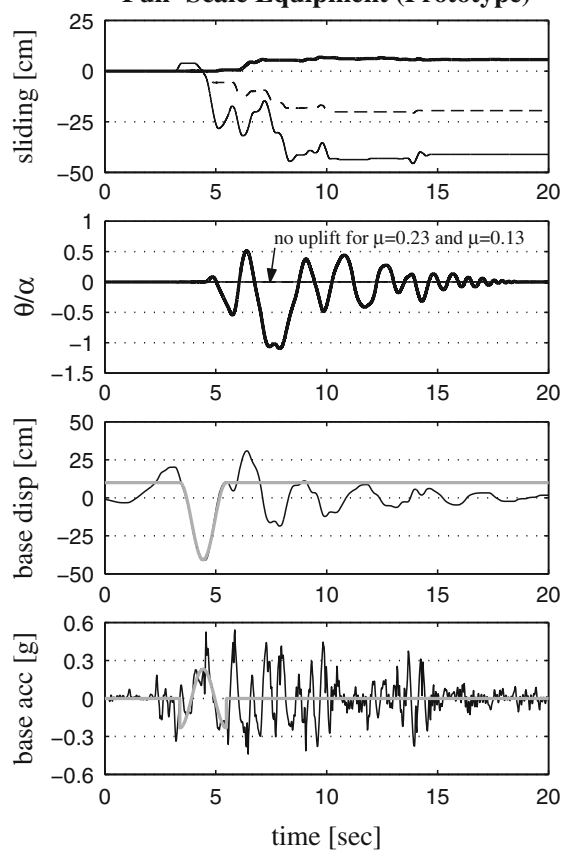

Fig. 7 Response of the FORMA incubator to the Loma Prieta, Los Gatos PC, FP Ground (2\% in 50years) motion. Left: Outcome of the shake table experiment on the quarter-scale wooden block model together with the table acceleration and displacement of the compressed (by a factor of 2) motion. Right: Computed response by Working Model simulations on the full-scale equipment prototype subjected to the uncompressed motion

the wooden model because the wooden model of the ASP refrigerator suffered damage that reduced its seismic stability. Figure 2 (bottom) shows a photograph of the damage on the corner of the wooden model and how this damage is also evident in the load-displacement curve. The reduced base (and therefore stability) due to the damage of the block's corners explains why, despite the large stockiness of the ASP refrigerator, the wooden model toppled in all cases, while the Working Model simulation predicted that the prototype topples in only 5 out of the 9 cases. More details on the experimental results on the wooden models and numerical simulation of full-scale equipment to $2 \%$ in 50 years are offered in the report by Konstantinidis and Makris (2005b).

The comparison between experimental and computed results demonstrate Working Model's ability to capture the experimentally observed behavior and confirm the validity of the analytical models. This provides us with confidence that the program can compute the seismic response of laboratory equipment for a wider range of friction coefficients and hazard-level base motions.

\section{Sliding response due to $2 \%$ in 50 years hazard motions}

When the values of $\mu$ from the quasi-static pull tests were used, the computed sliding and rocking time histories presented in the report by Konstantinidis and Makris (2005b) showed a response that was contrary to the primarily sliding response that was experimentally observed for the 10 and $50 \%$ in 50 years motions. In particular, the numerical simulations predicted 
equipment: FORMA incubator

motion: Loma Prieta, Corralitos, FP GROUND (2\% in 50 years)

Working Model 2D simulation legend:

$-\mu=0.68$, from Slow Pull Tests on wooden blocks
$-\mu=0.23$, from Slow Pull Tests on equipment
$-\mu=0.13$, from best fit of Shake Table Test results on equipment

Quarter-Scale Wooden Block (Model)
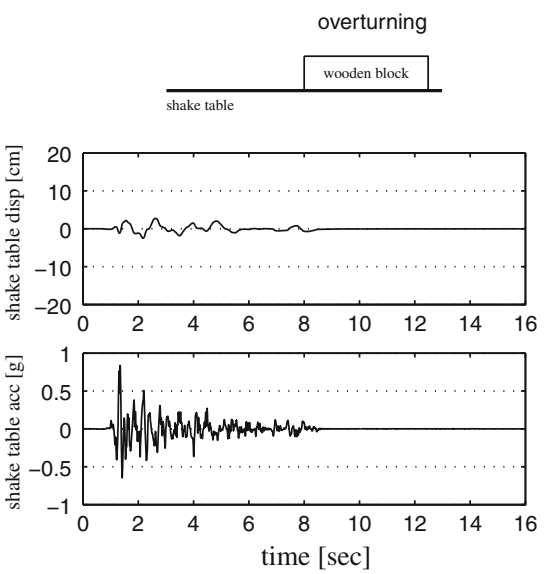

Full-Scale Equipment (Prototype)
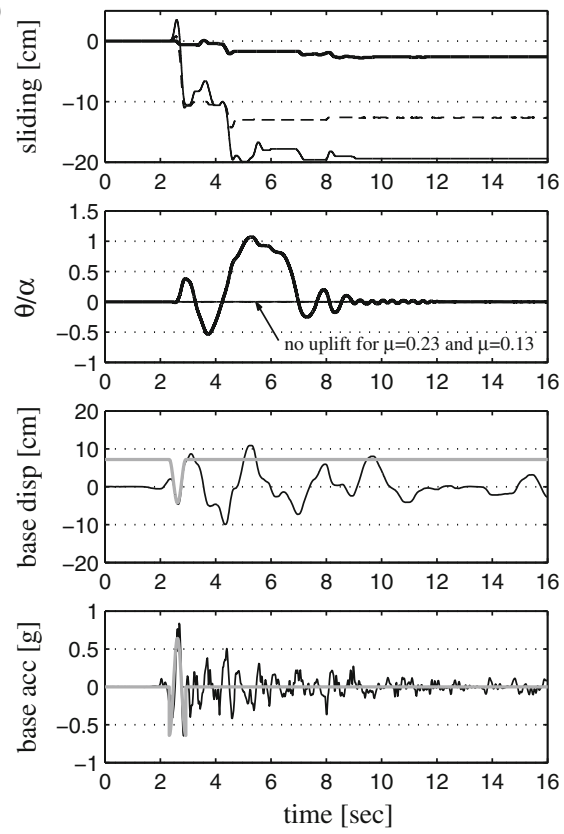

Fig. 8 Response of the FORMA incubator to the Loma Prieta, Corralitos, FP Ground ( $2 \%$ in 50 years) motion

equipment: Kelvinator refrigerator motion: Loma Prieta, Corralitos, FP GROUND (2\% in 50 years) Working Model 2D simulation legend:

$-\mu=0.68$, from Slow Pull Tests on wooden blocks
$-\mu=0.28$, from Slow Pull Tests on equipment
$-\mu=0.17$, from best fit of Shake Table Test results on equipment

Quarter-Scale Wooden Block (Model)
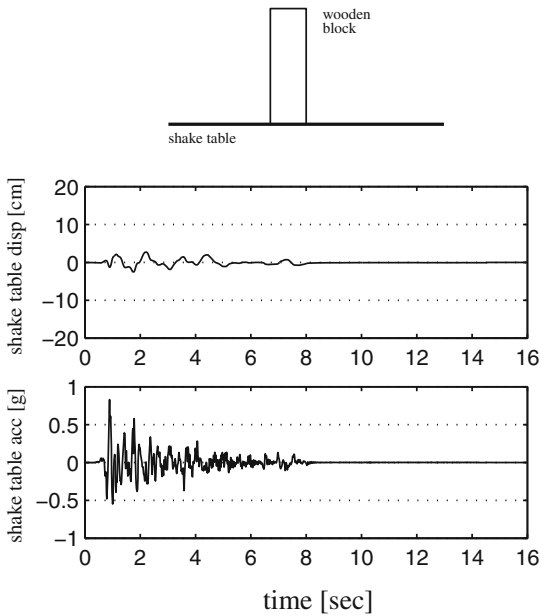

Full-Scale Equipment (Prototype)
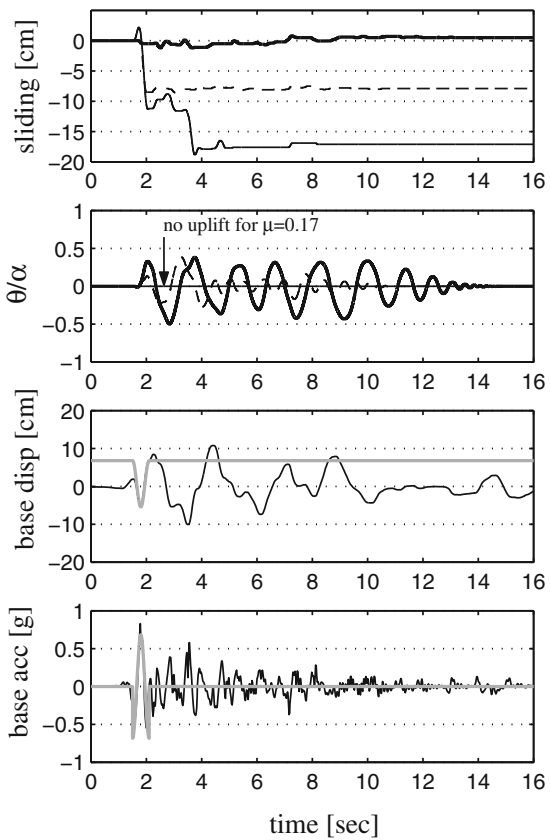

Fig. 9 Response of the KELVINATOR refrigerator to the Loma Prieta, Corralitos, FP Ground ( $2 \%$ in 50 years) motion 
that the KELVINATOR and ASP refrigerators experience large rotations-and even overturning in a few occasions. In light of the earlier observations where the KELVINATOR and ASP refrigerators in fact experienced very small rotations, it was concluded that the friction coefficients from the slow pull test were too large, and consequently they caused the equipment to engage in rocking during the numerical simulations. A considerably improved agreement between experimental and numerical results was observed when $\mu$ was reduced in the numerical simulations. As explained in Konstantinidis and Makris (2009), part of the reason for the necessary reduction is possibly pressure-dependence of $\mu$ that was not captured during the quasi-static pull tests. This would be similar to the pressure-dependent behavior of a teflon-steel interface observed by other investigators (Mokha et al. 1988; Constantinou et al. 1993). Moreover, a reduction in $\mu$ was necessary because the Working Model blockto-base interface is rigid-plastic (Coulomb), while the actual behavior is elastoplastic, where the elasticity originates from the flexibility of the equipment legs. Recent studies (Makris and Black 2004; Makris and Psychogios 2006) have shown that for the same value of $\mu$, the sliding displacement increases with increasing yield displacement. Consequently, given that in all three pieces of equipment the behavior at the sliding interface is elastoplastic (finite yield displacement), when a rigid-plastic model is adopted to capture the behavior, a smaller value of $\mu$ is needed.

The reduced values of $\mu$ were chosen so as to best-match the computed response to the experimentally observed one. For the present study, Working Model simulations for $2 \%$ in 50 years motions with the best-fitted friction coefficient values result in the predominant sliding response that is anticipated (plotted with solid black lines in Figs. 7, 8, 9). For this reason, the analysis presented in this paper uses the reduced (or best-fit) friction coefficients listed in Table III of Konstantinidis and Makris (2009), which is also repeated herein on the left column of Table 3 . Table 3 lists the $2 \%$ in 50 years input motions used with the Working Model simulations and the computed peak sliding displacement, $U_{\max }$, of the laboratory equipment.

\section{Regression analysis and fragility curves}

Since we are primarily concerned with sliding and not rocking, the parameters that govern the response of a sliding body become those that describe (a) the mechanical characteristics of the equipment-floor interface and (b) the kinematic characteristics of the base motion. As demonstrated in Konstantinidis and Makris (2009), the parameter that best characterizes the sliding resistance of the contact interface is the kinetic coefficient of friction $\mu$.

The impulsive character of near-fault ground motions can be adequately described by physically realizable trigonometric pulses. The input parameters of the model have an unambiguous physical meaning. The minimum number of parameters is two, which are either the acceleration amplitude, $a_{p}$, and duration, $T_{p}$, or the velocity amplitude, $v_{p}$, and duration, $T_{p}$ (Makris 1997; Makris and Chang 2000; Mavroeidis and Papageorgiou 2003). The third column of Table 3 lists the defining parameters of Type-B trigonometric pulses that approximate the predominant pulses of each of the $2 \%$ in 50 years motions used in this study. A Type-B pulse, which results in a forward-and-back ground displacement, is defined by $u_{g}^{\mathrm{B}}(t)=v_{p}\left[1-\cos \left(\omega_{p} t\right)\right] / \omega_{p}, 0 \leq t \leq T_{p}$ (Makris 1997). The velocity and acceleration histories are obtained by differentiating the expression for ground displacement, $u_{g}^{\mathrm{B}}(t)$. The acceleration and displacement histories of such pulses are plotted with heavy grey lines on the 
bottom two windows of Figs. 7, 8 and 9 (right) together with the corresponding earthquake records (black lines).

\subsection{Intensity measure and engineering demand parameter}

The estimation of the sliding response is cast in a probabilistic framework. The PEER-proposed PBEE methodology (Porter 2003) suggests the identification of an Intensity Measure (IM) and Engineering Demand Parameter (EDP). The choices for the IM and EDP emerge from dimensional analysis in conjunction with previously published results on the response of a sliding block (Newmark 1965; Barenblatt 1996; Konstantinidis and Makris 2005a,b). For a pulse-type excitation, the maximum sliding displacement $U_{\max }$ can be expressed as a function of independent variables $a_{p}, \omega_{p}$ and $\mu g$

$$
U_{\text {max }}=f\left(a_{p}, \omega_{p}, \mu g\right)
$$

where $\omega_{p}=2 \pi / T_{p}$ is the circular frequency of the pulse, or if an earthquake motion is approximated by a pulse, the circular frequency of the pulse that approximates the predominant pulse of the earthquake. The dependent variable $U_{\max }$ and independent variables $a_{p}$, $\omega_{p}$ and $\mu g$, involve only two dimensions, those of length, $L$, and time, $T$. The quantities of interest have dimensions

$$
\left[U_{\text {max }}\right]=L, \quad\left[a_{p}\right]=L T^{-2}, \quad\left[\omega_{p}\right]=T^{-1}, \quad[\mu g]=L T^{-2}
$$

Buckingham's Pi-Theorem states that a dimensionally homogeneous equation with a total of $k$ variables and $r$ reference dimensions, can be reduced to a relationship among $k-r$ independent dimensionless $\Pi$-products (Barenblatt 1996). Accordingly, in this case there are $4-2=2$ dimensionless products. Two obvious choices for $\Pi$-products are

$$
\Pi_{1}=\frac{U_{\max } \omega_{p}^{2}}{a_{p}}, \quad \Pi_{2}=\frac{\mu g}{a_{p}}
$$

and the two are related by a function

$$
\Pi_{1}=\phi\left(\Pi_{2}\right)
$$

For a rectangular acceleration pulse, this function is (Newmark 1965)

$$
\Pi_{1}=2 \pi^{2}\left(\frac{1}{\Pi_{2}}-1\right)
$$

For the case of trigonometric pulses, such as a Type-B pulse, the response is again described by Eq. (5) (Makris and Black 2003; Konstantinidis and Makris 2005b), and the form of the function $\phi$ is obtained numerically. Figure 3 plots with solid lines the response due to a rectangular acceleration pulse excitation and to a one-sine acceleration pulse excitation (i.e., a Type-A pulse, defined by $\left.u_{g}^{\mathrm{A}}(t)=v_{p}\left[\omega_{p} t-\sin \left(\omega_{p} t\right)\right] /\left(2 \omega_{p}\right), 0 \leq t \leq T_{p}\right)$. The dimensionless displacement $\Pi_{1}=U_{\max } \omega_{p}^{2} / a_{p}$ is plotted on a logarithmic scale in order to illustrate the relative strengths of the two types of pulses. The closed-form solution due to the rectangular pulse is given by Eq. (6), while the solution due to the one-sine pulse is obtained numerically using standard ODE solvers available in MATLAB (2002).

The choices for IM and EDP used in this study fall naturally from Eqs. (4) and (6), and are

$$
\mathrm{IM}=\frac{\mathrm{PTA}}{\mu g}-1
$$


and

$$
\Delta=\frac{U_{\max } \omega_{p}^{2}}{\text { PTA }}
$$

The chosen EDP will henceforth be designated $\Delta$. The last two columns of Table 3 list the IM and EDP. Note from Eqs. (4), (6) and (7) that the IM chosen is exactly the quantity in parenthesis in Eq. (6) except that the IM uses for simplicity PTA instead of $a_{p}$. The two values are expected to be close.

\subsection{The EDP as a lognormal random variable; regression of numerical simulation data}

Figure 10 (bottom) plots the Engineering Demand Parameter, $\Delta=U_{\max } \omega_{p}^{2} / \mathrm{PTA}$, as a function of the Intensity Measure, IM $=$ PTA $/ \mu g-1$, obtained from Working Model simulations on the three pieces of equipment subjected to the $2 \%$ in 50 years hazard level motions listed in Table 3. It is obvious that the data exhibits considerable scatter, which suggests that $\Delta$ has to be treated as a random variable. When a random variable, $\Delta$, expresses a quantity that is only positive $(\delta>0)$, it is common to assume that the variable is lognormally distributed. In this study we hypothesize that the EDP is lognormally distributed, and we test this hypothesis against the experimental results. The reason behind the name lognormal is that the lognormally distributed variable $\Delta$ is related to a normally distributed variable $X$ by $X=\ln \Delta$. Note that $\Delta$ attains only positive values, $\delta>0$, while the corresponding $X$ variable is unrestricted, $-\infty<x<\infty$. It is assumed that $\Delta$ takes the form

$$
\Delta=\zeta\left(\frac{\mathrm{PTA}}{\mu g}-1\right)^{\eta} Z=\zeta \mathrm{IM}^{\eta} Z
$$

where $\zeta$ and $\eta$ are regression parameters and $Z$ is a lognormally distributed random variable. Another important feature of the scatter in Fig. 10 (bottom) is that it increases with increasing IM (funneling effect), i.e., the variance is non-constant. However, the logarithmic transformation

$$
X=\ln \Delta=\ln \zeta+\eta \ln \mathrm{IM}+\ln Z
$$

stabilizes the variance (homoscedasticity), as seen on the top left plot of Fig. 10, and therefore ordinary least-squares can be applied to estimate the parameters $\zeta$ and $\eta$. We obtain

$$
\Delta=0.370\left(\frac{\mathrm{PTA}}{\mu g}-1\right)^{0.953} Z=0.370 \mathrm{IM}^{0.953} Z
$$

The lognormally distributed variable has the probability density function

$$
f_{\Delta}(\delta)=\frac{1}{\sqrt{2 \pi} \sigma_{X} \delta} e^{-\frac{1}{2}\left(\frac{\ln \delta-m_{X}}{\sigma_{X}}\right)^{2}}, \quad \delta>0
$$

where the two defining parameters $m_{X}$ and $\sigma_{X}$ of the distribution are in fact the mean and standard deviation of the corresponding normally distributed variable $X$ (Crow et al. 1988). The cumulative distribution function of $\Delta$ is given by

$$
F_{\Delta}(\delta)=\int_{0}^{\delta} \frac{1}{\sqrt{2 \pi} \sigma_{X} \delta^{\prime}} e^{-\frac{1}{2}\left(\frac{\ln \delta^{\prime}-m_{X}}{\sigma_{X}}\right)^{2}} d \delta^{\prime}
$$


Fig. 10 Top: The normal random variable $X=\ln \Delta$ and the linear fit which gives its mean $m_{X}$.

Bottom: The associated lognormal random variable $\Delta$, together with the power-law fit for its mean $m_{\Delta} . \sigma_{X}$ is constant, while $\sigma_{\Delta}$ increases with IM
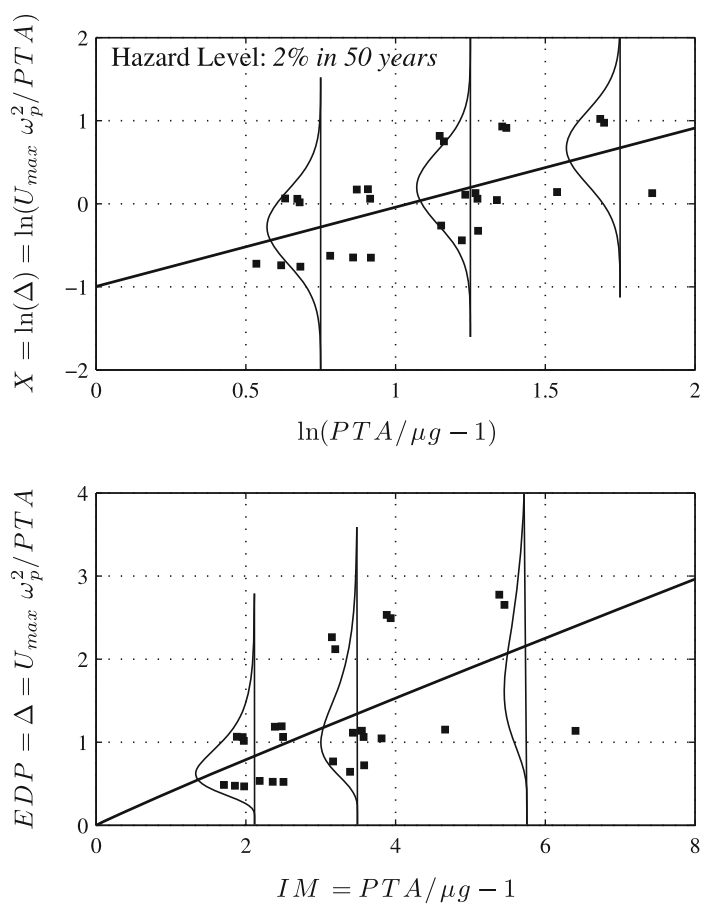

Using the substitution $u=\left(\ln \delta^{\prime}-m_{X}\right) / \sigma_{X}$, we obtain

$$
F_{\Delta}(\delta)=\int_{-\infty}^{\left(\ln \delta-m_{X}\right) / \sigma_{X}} \frac{1}{\sqrt{2 \pi}} e^{-\frac{u^{2}}{2}} d u=\Phi\left(\frac{\ln \delta-m_{X}}{\sigma_{X}}\right)
$$

where $\Phi$ is the cumulative distribution function of a standard normal variable (i.e., mean of zero and standard deviation of one).

Note from Eq. (10) that the mean of $X$ is $m_{X}(\mathrm{IM})=\ln \left(\zeta \mathrm{IM}^{\eta}\right)$. Also, the standard deviation of $X$ can be estimated from

$$
\sigma_{X}=\sigma_{\ln Z}=\sqrt{\frac{1}{n-1} \sum_{i=1}^{n}\left(\ln \frac{\delta_{i}}{\zeta \mathrm{IM}_{i}^{\eta}}\right)^{2}}
$$

where $n$ is the sample size. Using the results of the 27 Working Model simulations on the full-scale equipment (Table 3), we obtain $\sigma_{X}=0.445$. The mean $m_{\Delta}$ (IM) and standard deviation $\sigma_{\Delta}$ (IM) of $\Delta$ can be shown to be related to the mean $m_{X}$ (IM) and standard deviation $\sigma_{X}$ of the normal variable $X$ through

$$
m_{\Delta}(\mathrm{IM})=e^{m_{X}(\mathrm{IM})+\frac{\sigma_{X}^{2}}{2}}=\zeta \mathrm{IM}^{\eta} e^{\frac{\sigma_{X}^{2}}{2}}
$$

and

$$
\sigma_{\Delta}(\mathrm{IM})=e^{m_{X}(\mathrm{IM})+\frac{\sigma_{X}^{2}}{2}} \sqrt{e^{\sigma_{X}^{2}}-1}=\zeta \mathrm{IM}^{\eta} e^{\frac{\sigma_{X}^{2}}{2}} \sqrt{e^{\sigma_{X}^{2}}-1}
$$


Fig. 11 The empirical cumulative distribution function, $F_{\Delta}^{*}$, and the theoretical cumulative distribution function $F_{\Delta}$ of its hypothesized lognormal distribution

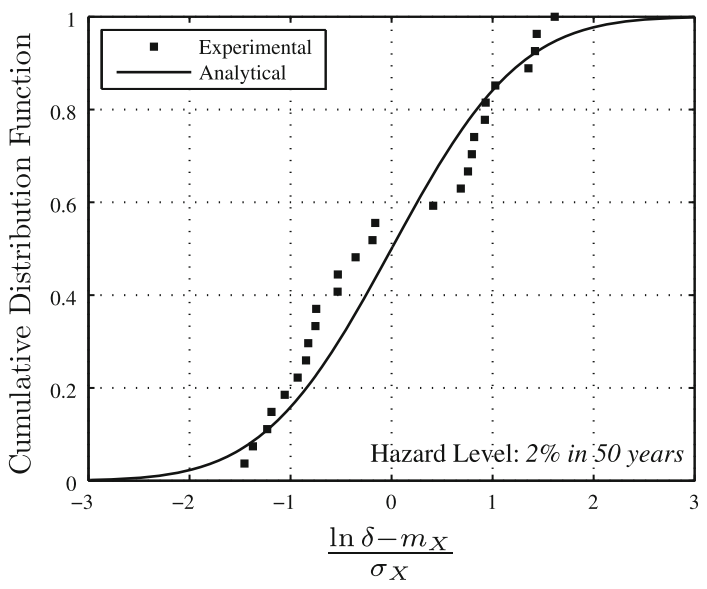

Figure 10 (top) shows how $X$ is distributed with a constant standard deviation at three different IM values, while Fig. 10 (bottom) shows how $\Delta$ is distributed with an increasing $\sigma_{\Delta}(\mathrm{IM})$.

In order to not reject the hypothesis that $\Delta$ has a lognormal distribution, the KolmogorovSmirnov goodness-of-fit test is performed (Scheaffer and McClave 1995). Figure 11 plots the empirical cumulative distribution function, $F_{\Delta}^{*}=i / n$. Together is plotted the cumulative distribution function of the hypothesized lognormal distribution, $F_{\Delta}=\Phi\left(\left(\ln \delta-m_{X}\right) / \sigma_{X}\right)$. The maximum distance between the distribution functions presented in Fig. 11 is

$$
S=\max _{\delta}\left|F_{\Delta}^{*}(\delta)-F_{\Delta}(\delta)\right|=0.161
$$

Tables for the Kolmogorov-Smirnov test (Daniel 1990) provide $S_{c r}$ for $n=27$ and various significance levels, $a$,

$$
S_{c r}(a, n=27)= \begin{cases}0.305, & a=0.01 \\ 0.284, & a=0.02 \\ 0.254, & a=0.05 \\ 0.229, & a=0.10 \\ 0.200, & a=0.20\end{cases}
$$

Since $S<S_{c r}$, the hypothesis that $\Delta$ is lognormally distributed is not rejected.

\subsection{Fragility curves}

Fragility is viewed as a conditional probability of failure. For the problem at hand, where we want to characterize the seismic response of heavy laboratory equipment in a probabilistic framework, we define fragility as the probability $P_{f}$ that the EDP, $\Delta=U_{\text {max }} \omega_{p}^{2} / \mathrm{PTA}$, for a piece of equipment will exceed a certain threshold (capacity), $c$, given the IM. For the lognormally distributed random variable $\Delta$,

$$
P_{f} \equiv P(\Delta>c \mid \mathrm{IM})=1-P(\Delta<c \mid \mathrm{IM})=1-F_{\Delta}(c)
$$

Substituting Eq. (14) and $m_{X}(\mathrm{IM})=\ln \left(\zeta \mathrm{IM}^{\eta}\right)$ into Eq. (19) gives

$$
P_{f}=1-\Phi\left(\frac{\ln c-m_{X}(\mathrm{IM})}{\sigma_{X}}\right)=1-\Phi\left(\frac{1}{\sigma_{X}} \ln \frac{c}{\zeta \mathrm{IM}^{\eta}}\right)
$$


Fig. 12 Fragility curves for four different $c$ 's

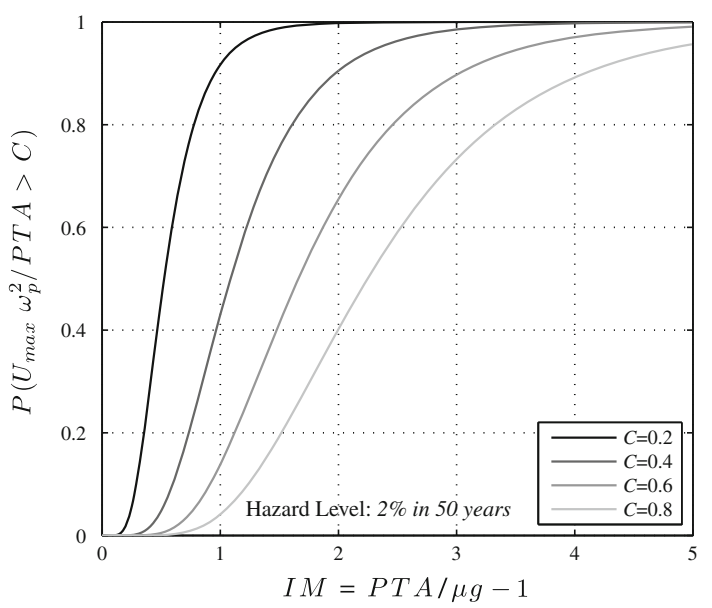

where $\Phi$ is the standard normal cumulative distribution function, and

$$
\sigma_{X}=0.445, \quad \zeta=0.370, \quad \eta=0.953
$$

Once the $\sigma_{X}$ is estimated from Eq. (15) and the regression parameters $\zeta$ and $\eta$ are obtained from ordinary least-squares fit on Eq. (10), the fragility curves can be generated using Eq. (20) for different capacities $c$. Figure 12 shows fragility curves for four values of capacity $c$ $(0.2,0.4,0.6$, and 0.8$)$. Typically, fragility curves are plotted against the EDP. In this study, however, they are plotted against the IM, as this makes their use more direct. Konstantinidis and Makris (2009) offer an example of how to use such fragility curves.

\section{Conclusions}

This paper investigates the response of freestanding laboratory equipment subjected to strong earthquake shaking ( $2 \%$ in 50 years hazard level) via model testing. The shake table displacement capacity could not accommodate the ground and floor motions with $2 \%$ probability of being exceeded in 50 years. Instead, tests were conducted on quarter-scale wooden block models of the equipment so that the table displacements were reduced by a factor of four. The large friction coefficient of the wooden block-to-base interface caused the wooden blocks to rock and overturn rather than slide. The results from the shake table tests on the wooden models were compared to results from numerical simulations produced by Working Model in order to evaluate the ability of the software to reliably capture the overturning potential of equipment. Working Model's accuracy in predicting overturning and estimating sliding displacements gave us confidence on its overall ability to compute the response of a piece of equipment for different friction coefficients. Numerical simulations for the full-scale equipment under $2 \%$ in 50 years motions resulted in a response that is dominated by sliding, with maximum sliding displacements up to $70 \mathrm{~cm}$.

A physically motivated Intensity Measure, IM, and the associated Engineering Demand Parameter, EDP, for the $2 \%$ in 50 years hazard level were identified with the help of dimensional analysis. Relationships for the mean and standard deviation of the EDP in terms of the IM were developed. Finally, the paper presents ready-to-use fragility curves, which give the probability that the EDP will exceed a specified threshold $c$ for a given IM. 
Acknowledgments From the Earthquake Simulator Laboratory at the Pacific Earthquake Engineering Research (PEER) Center, UC Berkeley, where the shake table experiments were conducted, we greatly appreciate the technical assistance of Don Clyde, Wes Neighbour, and David Maclam. We would also like to thank our PEER colleagues for their collaboration. We are very grateful for their continual feedback throughout the course of this study. This work was supported primarily by the Earthquake Engineering Research Centers Program of the National Science Foundation under award number EEC-9701568 through the Pacific Earthquake Engineering Research Center (PEER).

Open Access This article is distributed under the terms of the Creative Commons Attribution Noncommercial License which permits any noncommercial use, distribution, and reproduction in any medium, provided the original author(s) and source are credited.

\section{References}

Barenblatt GI (1996) Scaling, self-similarity, and intermediate asymptotics. Cambridge University Press, Cambridge

Comerio MC (ed) (2005) PEER testbed study on a laboratory building: exercising seismic performance assessment. Report No. PEER 2005-12, Pacific Earthquake Engineering Research Center. University of California, Berkeley

Constantinou MC, Tsopelas P, Kim YS, Okamoto S (1993) NCEER-Taisei Corporation research program on sliding seismic isolation systems for bridges: experimental and analytical study of a friction pendulum system (FPS). Technical Report NCEER-93-0020, National Center for Earthquake Engineering Research. State University of New York at Buffalo, New York

Crow EL, Shimizu K (eds) (1988) Lognormal distributions. Marcel Dekker, New York

Daniel WW (1990) Applied nonparametric statistics, 2nd edn. PWS-KENT, Boston

Hutchinson TC, Chaudhuri SR (2006) Bench-shelf system dynamic characteristics and their effects on equipment and contents. Earthq Eng Struct Dyn 35(13):1631-1651

Konstantinidis D, Makris N (2005a) Seismic response analysis of multidrum classical columns. Earthq Eng Struct Dyn 34(10):1243-1270

Konstantinidis D, Makris N (2005b) Experimental and analytical studies on the seismic response of freestanding and anchored laboratory equipment. Report No. PEER 2005-07, Pacific Earthquake Engineering Research Center. University of California, Berkeley

Konstantinidis D, Makris N (2009) Experimental and analytical studies on the response of freestanding laboratory equipment to earthquake shaking. Earthq Eng Struct Dyn 38(6):827-848

Lee TH, Mosalam KM (2005) Seismic demand sensitivity of reinforced concrete shear-wall building using FOSM method. Earthq Eng Struct Dyn 34(14):1719-1736

Lopez Garcia D, Soong TT (2003a) Sliding fragility of block-type non-structural components. Part 1: unrestrained components. Earthq Eng Struct Dyn 32(1):111-129

Lopez Garcia D, Soong TT (2003b) Sliding fragility of block-type non-structural components. Part 2: restrained components. Earthq Eng Struct Dyn 32(1):131-149

Makris N (1997) Rigidity-plasticity-viscosity: can electrorheological dampers protect base-isolated structures from near-source ground motions? Earthq Eng Struct Dyn 26(5):571-591

Makris N, Black CJ (2003) Dimensional analysis of inelastic structures subjected to near-fault ground motions. Report No. EERC 2003-05, Earthquake Engineering Research Center. University of California, Berkeley

Makris N, Black CJ (2004) Dimensional analysis of rigid-plastic and elastoplastic structures under pulse-type excitations. J Eng Mech (ASCE) 130(9):1006-1018

Makris N, Chang SP (2000) Response of damped oscillators to cycloidal pulses. J Eng Mech (ASCE) 126(2):123-131

Makris N, Konstantinidis D (2003a) The rocking spectrum and the limitations of practical design methodologies. Earthq Eng Struct Dyn 32(2):265-289

Makris N, Konstantinidis D (2003b) The rocking spectrum, existing design guidelines, and a scale invariance. In: Proceedings of the fédération internationale du Béton symposium, Athens, 6-9 May 2003

Makris N, Psychogios T (2006) Dimensional response analysis of yielding structures with first-mode dominated response. Earthq Eng Struct Dyn 35(10):1203-1224

Makris N, Roussos Y (2000) Rocking response of rigid blocks under near-source ground motions. Géotechnique 50(3):243-262

MATLAB (2002) High-performance language software for technical computing. The MathWorks, Inc., Natick 
Mavroeidis GP, Papageorgiou AS (2003) A mathematical representation of near-fault ground motions. Bull Seismol Soc Am 93(3):1099-1131

Mokha A, Constantinou MC, Reinhorn AM (1988) Teflon bearings in aseismic base isolation: experimental studies and mathematical modeling. Technical Report NCEER-88-0038, National Center for Earthquake Engineering Research. State University of New York at Buffalo, New York

Newmark NM (1965) Effects of earthquakes on dams and embankments. Fifth rankine lecture. Géotechnique 15:139-160

Porter KA (2003) An overview of PEER's performance-based earthquake engineering methodology. In: Proceedings of the ninth international conference on applications of statistics and probability in civil engineering (ICASP9), San Francisco 6-9 July 2003

Scheaffer RL, McClave JT (1995) Probability and statistics for engineers, 4th edn. Duxbury Press, Belmont

Shao Y, Tung CC (1999) Seismic response of unanchored bodies. Earthq Spectra 15(3):523-536

Somerville PG (2001) Ground motion time histories for the UC Lab building. URS Corporation, Pasadena

Wen YK (1975) Approximate method for nonlinear random vibration. J Eng Mech Div (ASCE) 101(EM4): 389-401

Wen YK (1976) Method for random vibration of hysteretic systems. J Eng Mech Div (ASCE) 102(EM2):249263

Working Model (2000) User's manual. MSC. Software Corporation, San Mateo

Yim CS, Chopra AK, Penzien J (1980) Rocking response of rigid blocks to earthquakes. Earthq Eng Struct Dyn 8(6):565-587

Younis C, Tadjbakhsh G (1984) Response of sliding rigid structure to base excitation. J Eng Mech (ASCE) 110(3):417-432

Zhang J, Makris N (2001) Rocking response of free-standing blocks under cycloidal pulses. J Eng Mech (ASCE) 127(5):473-483 